\title{
Editorial
}

\section{Neoadjuvant chemotherapy: a standard treatment for locally advanced gastric cancer in the near future?}

\author{
JUNICHI SAKAMOTO \\ Department of Epidemiological and Clinical Research Management, Kyoto University, Graduate School of Medicine Yoshida-Konoe-cho, \\ Sakyo-ku, Kyoto 606-8501, Japan
}

In this issue of Gastric Cancer, Ott and colleagues from the Technichen Universitat in Munich, Germany, present a 49-patient prospective Phase II study of the efficacy of cisplatin-high-dose folinic acid-high-dose 5fluorouracil (PLF) chemotherapy [1], delivered preoperatively for locally advanced $\left(\mathrm{cT}_{3,4}, \mathrm{~N}_{\mathrm{x}}, \mathrm{M}_{\mathrm{x}}\right)$ gastric cancer $[1,2]$. The primary endpoint of the study was the clinical response of the primary lesion to preoperative therapy.

The goal of surgery for gastric carcinoma is a curative resection that involves removal of all gross cancer and regional lymph node groups, without leaving any macroscopically visible cancer lesions. Patients with locally advanced disease who have undergone an $\mathrm{R}_{0}$ resection constitute the best prognostic group, with an estimated 5 -year survival of $25 \%$ in Western countries and approximately $30 \%$ in Japan [2].

Neoadjuvant chemotherapy for gastric cancer aims to down-stage patients, improve curative resectability of locally advanced disease, and eventually increase patient survival. Several reasonable rationales justify the use of adjuvant chemotherapy as an induction step in multidisciplinary treatment. Introducing chemotherapy in an early phase may, theoretically, facilitate delivery of drugs towards the primary lesion, without impaired vascularization. It is also considered effective in reducing occult micrometastases, in which cell growth fraction is high and heterogeneity is relatively low. In addition, major surgery such as total gastrectomy delays the start of postoperative systemic chemotherapy by a month or more, giving microscopically residual diseases an opportunity to proliferate. Neoadjuvant chemotherapy is also advantageous, as it can provide important information for the postoperative use of che-

Offprint requests to: J. Sakamoto

Received: June 30, 2003 / Accepted: July 3, 2003 motherapeutic agents by evaluating the response of the resected primary tumor.

Ott et al. carefully conducted a prestudy diagnosis with less invasive endoscopic ultrasound (US) and computed tomogrphy (CT) scans. Forty two of the 49 patients received at least one course of PLF, and 38 patients successfully received two cycles as scheduled. The drug was tolerated in $80 \%$ of the enrolled patients and the compliance for the treatment schedule appeared feasible.

Did neoadjuvant chemotherapy down-stage patients? Clinical response evaluation showed a partial response in $26 \%$ of patients and a minor response in $48 \%$. Postoperative UICC/AJCC pTNM classification staged 4 patients as ypT1 and 23 patients as ypT2. Compared with the pretherapeutic staging based on endoscopic ultrasound (EUS), 30 patients had lower pT categories. Based on these results, this neoadjuvant chemotherapy regimen obviously did down-stage patients.

Did neoadjuvant chemotherapy improve curative resectability? Thirty two of the 42 eligible patients underwent a complete resection. The ratio of complete curative $\mathrm{R}_{0}$ resection was not clearly cited in the report, but it should be almost identical to the above numbers, given that the total number of $\mathrm{pN} 0+\mathrm{pN} 1+\mathrm{pN} 2$ patients was 35, and extended D2 lymphadenectomy was performed on all eligible patients. Although we must be prudent in determining whether neoadjuvant chemotherapy improved curative resectability, the estimated percentage of $\mathrm{R}_{0}$ resections for the eligible $\mathrm{cT}_{3-4}$ patients might be an acceptable figure both in Western countries and in Japan.

Did neoadjuvant chemotherapy improve prognosis? The estimated overall 5-year survival rate was about $40 \%$, and in patients who underwent curative $R_{0}$ resection, it was approximately 50\%. Among 11 clinically responding patients, the 5-year survival rate was nearly $90 \%$, but for 31 nonresponders it was less than $20 \%$. Despite the preexisting goal to achieve a $10 \%-15 \%$ 
pathologically confirmed complete response [2], where only those patients achieving complete response would have a survival advantage [3], an improved 5-year survival rate was demonstrated even in the partial responders. Thus, according to the results from Ott et al., neoadjuvant chemotherapy also improved prognosis.

The implementation of neoadjuvant therapies in the treatment of gastric cancer certainly requires meticulous and accurate pretreatment diagnosis and evaluation of the preoperative treatment by surgeons. The surgeon must be confident that patients are optimally staged and eligible for the induction treatment and also be aware of the effects and toxicities of preoperative chemotherapy.

To that end, several new and more elaborate diagnostic methodologies have recently been developed. Although invasive, the combined use of laparoscopy and EUS was shown to enhance accurate staging [4]. Sentinel lymph node biopsies have been used to determine pathologic outcomes for patients receiving neoadjuvant chemotherapy for breast cancer [5] and could be an effective tool to accurately evaluate lymph node metastasis before surgery for gastric cancer. As a noninvasive technique, a new diagnostic method using color Doppler has been developed to examine vascularity and microvessel density in the primary and metastatic site [6]. The use of various enzymatic markers, such as thymidylate synthase, dehydropyrimidine dehydrogenase, and matrix metalloproteinase, together with molecular markers, such as microsatellite instability, loss of heterogeneity, or p53 mutation [7], has been exploited for pretrial selection, treatment monitoring, and therapeutic endpoint guidance.

The identification of an effective chemotherapy regimen and optimal treatment schedule for locally advanced disease has been another important issue in neoadjuvant treatment for gastric cancer. To date, there have been many anecdotal case reports and a number of Phase II neoadjuvant chemotherapy trials published for potentially resectable gastric cancer in both Japan and Western countries [8-10]. Various regimens and schedules have been tested in those studies, including CDDP/ 5FU, CPT11/CDDP, UFT/CDDP, UFT, S1, S1/CDDP, ECF, EC/Doxorubicin, PELF, PELF/Taxotere, and $\mathrm{PLF} /$ Taxol. These studies have shown that for preoperative therapy to potentially impact gastric cancer, neoadjuvant treatment should be based on a combination of effective agents that can be administered safely for limited periods of time at maximum tolerated doses.

What is our next goal? Focus should be placed on the examination of new drugs, preoperative radiotherapy, and new combinations of these modalities in well designed and carefully conducted Phase II studies. However, evaluation of an adequately sized randomized Phase III trial is indispensable before any firm conclu- sion can be established. Several Phase III trials are currently accruing patients in Britain (pre- and postoperative ECF), France (CDDP/5FUCI), Switzerland, and Italy (CDDP /5FUCI/Docetaxel), and by EORTC (CDDP/5FU). At the American Society of Clinical Oncology (ASCO) meeting held in June 2003, a report on the British trial (MAGIC) was given by Allum et al. [11]. In this randomized Phase III trial, which included a total of 503 locally advanced gastric cancer patients, 250 patients were assigned to receive preoperative chemotherapy-surgery-postoperative chemotherapy (CSC group), and 253 received surgery alone (S group). According to their report, curative resection rates were $79 \%$ in the CSC group and $69 \%$ in the S group, and neoadjuvant treatment was shown to be significantly superior to surgery alone, with a $P$ value of 0.018 . As far as the prognosis of patients is concerned, the survival hazard ratio was $0.80(0.63-1.01, P=0.063)$ and the progression free survival hazard ratio was $0.70(0.56-$ $0.88, P=0.002)$. This result from the British randomized study is of potential value, as it has confirmed the significant effect of neoadjuvant chemotherapy for locally advanced gastric cancer in a prospective, single, large randomized trial that has accrued an optimal number of patients to evaluate its efficacy.

As indicated by both Ott's report and the report from the British randomized Phase III trial, there is now convincing evidence that neoadjuvant chemotherapy down-stages patients, improves resectability, and also improves the prognosis of locally advanced gastric cancer, with tolerable and manageable toxicity and without increasing the mortality or morbidity of surgery. In Japan, a study conducted by the Japan Clinical Oncology Group (JCOG) is currently underway to appropriately evaluate the role of chemotherapy in locally advanced gastric cancers.

The goal of these studies of neoadjuvant chemotherapy is to discover safer and more effective treatment modalities. While there has been some reluctance to adopt such adjunctive and cumbersome treatment, given these emerging findings, physicians must overcome their reservations and establish a new standard of treatment for locally advanced gastric cancer in the very near future.

\section{References}

1. Ott K, Sendler A, Becker K, Dittler H-J, Helmberger H, Busch $\mathrm{R}$, et al. Neoajuvant chemotherapy with cisplatin, 5-FU, and leucovorin (PLF) in locally advanced gastric cancer: a prospective phase II study. Gastric Cancer 2003;6:159-67.

2. Lowy AM, Mansfield PF, Leach SD, Pazdur R, Dumas P, Ajani JA. Response to neoadjuvant chemotherapy best predicts survival after curative resection of gastric cancer. Ann Surg 1999;229: 303-8. 
3. Swisher SG, Holmes EC, Hunt KK, Doty JE, Zinner MJ, McFadden DW. The role of neoadjuvant therapy in surgically resectable esophageal cancer. Arch Surg 1996;131:819-24.

4. Ajani JA, Mansfield PF, Lynch PA, Pisters PW, Feig B, Dumas P, et al. Enhanced staging and all chemotherapy preoperatively in patients with potentially resectable gastric carcinoma. J Clin Oncol 1999;17(8):2403-11.

5. Bland KI. Utilization of sentinel lymph node mapping to determine pathologic outcomes for patients receiving neoadjuvant chemotherapy for locally advanced breast cancer. Ann Surg Oncol 2002;9(3):217-9.

6. Chen CN, Cheng YM, Lin MT, Hsieh FJ, Lee PH, Chang KJ. Association of color Doppler vascularity index and microvessel density with survival in patients with gastric cancer. Ann Surg 2002;235(4):512-8.

7. Ott K,Vogelsang H, Mueller J, Becker K, Muller M, Fink U. Chromosomal instability rather than p53 mutation is associated with response to neoadjuvant cisplatin-based chemotherapy in gastric carcinoma. Clin Cancer Res 2003;9(6):2307-15.
8. Cascinu S, Graziano F, Barni S, Labianca R, Comella G, Casaretti $\mathrm{R}$, et al. A phase II study of sequential chemotherapy with docetaxel after the weekly PELF regimen in advanced gastric cancer. A report from the Italian group for the study of digestive tract cancer. Br J Cancer 2001;84(4):470-4.

9. Gallardo-Rincon D, Onate-Ocana LF, Calderillo-Ruiz G. Neoajuvant chemotherapy with P-ELF (cisplatin, etoposide, leucovorin, 5-fluorouracil) followed by radical resection in patients with initially unresectable gastric adenocarcinoma: a phase II study. Ann Surg Oncol 2000;7(1):45-50.

10. Takahashi S, Kinoshita T, Konishi M, Nakagouri T, Inoue K, Ono M, et al. Phase II study of sequential high-dose methotrexate and fluorouracil combined with doxorubicin as a neoadjuvant chemotherapy for scirrhous gastric cancer. Gastric Cancer 2001; 4(4):192-7.

11. Allum W, Cunningham D, Weeden S. Perioperative chemotherapy in operable gastric and lower oesophageal cancer: a randomized, controlled trial (the MAGIC trial, ISRCTN 9379371). Proc ASCO 2003;22:249 